\title{
Laparoscopia oficinal bajo anestesia local y sedación consciente
}

\author{
Jaime Saavedra S, MD
}

Recibido: Mayo $11 / 2000$

Revisado: Mayo $19 / 2000$

Aceptado: Agosto $11 / 2000$

\section{RESUMEN}

La laparoscopia bajo anestesia local traza su concepción muy cercanamente con el desarrollo de la laparoscopia misma, inicialmente ésta se implementó como un procedimiento bajo anestesia local y no como un procedimiento con anestesia general. Su desarrollo ha sido acelerado colocándose en el primer plano de la laparoscopia, debido a las preocupaciones que generaron los altos costos del procedimiento con anestesia general.

La micro laparoscopia bajo anestesia local es uno de los avances recientes de la cirugía mismamente invasiva, que combina dos técnicas, la primera es uso de laparoscopios de un calibre menor de $2 \mathrm{~mm}$. Y la segunda a realización del procedimiento laparoscópico en sitios diferentes al quirófano convencional.

La laparoscopia bajo anestesia local está indicada para procedimientos diagnósticos y procedimientos quirúrgicos simples.

El requerimiento más importante para el éxito de la laparoscopia bajo anestesia local es la determinación del paciente de que se le realice el procedimiento bajo esta técnica

PALABRAS CLAVES: Laparoscopia, anestesia local, micro laparoscopia.

\section{SUMMARY}

Laparoscopy under local anesthesia goes by the hand with laparoscopys origin, at the begging ibis was introduce as a procedure under because of the problems generated by the high costs of the procedure at different with general anesthesia.

Micro laparoscopy under local anesthesia, is one of the recent advances of the minimally invasive surgery, that combines two different techniques, the fist one is the use of laparoscopes with a size that is less than $2 \mathbf{m m}$., and the second one is the realization of the laparosocopic procedure a different places of the operating theater.

The laparoscopy under local anesthesia is used for diagnostic procedures and for simple operating theater procedures.

The most important requeriment for the success of a laparoscopy under local anesthesia, is the patients determination for taking this procedure under this technique.

KEY WORDS: Laparoscopy, local anesthesia, micro laparoscopy.

La práctica de la ginecología quirúrgica moderna ha sido revolucionada con el advenimiento de la laparoscopia, la cual ha tenido avances progresivos desde el simple trabajo diagnóstico hasta la realización de procedimientos quirúrgicos complejos en la actualidad.

La micro laparoscopia bajo anestesia local es uno de los recientes avances en la cirugía mínimamente invasiva, que combina dos técnicas:

La primera es el uso de instrumental y endoscopios de calibre pequeño a menudo de menos de $2 \mathrm{~mm}$.
La segunda es realizar la laparoscopia bajo anestesia local, en sitios no tradicionales (ejemplo: no sala de operaciones) tales como sala para procedimientos de un hospital o clínica o una oficina para examen médico.

Cada una de estas innovaciones se puede realizar individualmente y a la vez proporcionar beneficios a los médicos y pacientes. Por ejemplo, la micro laparoscopia se puede realizar en un quirófano bajo anestesia general. De igual manera, los equipos tradicionales de laparoscopia se pueden utilizar para realizar procedimientos bajo anestesia

Profesor titular. Departamento de Obstetricia y Ginecología. Universidad del Valle - Cali Colombia.

Jefe del Servicio de Medicina Reproductiva. Hospital Universidad del Valle - Cali.

Presidente Asociación Colombiana de Médicos Endoscopistas.

Director General de la Clínica de Cirugía Endoscópica y Láser, Centro Médico Los Andes, Cali-Valle del Cauca.

Director General del Centro de Biomedicina Reproductiva - FECUNDAR. Cali-Valle del Cauca. 
local y en sitios quirúrgicos no convencionales. Juntamente estas dos tecnologías crean un sinergismo que da origen a nuevas ideas que permiten modificaciones en técnica $y$ en procedimientos laparoscópicos.

La técnica de la laparoscopia oficinal bajo anestesia local encaja perfectamente para satisfacer la presión general que ejercen las empresas prestadoras de salud cuando evalúan calidad versus costos.

Es probable que esta técnica pronto llegará a ser parte de los métodos utilizados por el ginecólogo para el diagnóstico y una herramienta más en su armamentario quirúrgico.

Las ventajas de la micro laparoscopia bajo anestesia local se pueden separar dentro de aquellas realizadas por el médico, el paciente y las instituciones prestadoras de salud. Tabla 1. A, B, C.

No obstante, la micro laparoscopia oficinal bajo anestesia local tiene varias desventajas inherentes al uso de endoscopios pequeños y a equipo frágil, la eliminación de la anestesia general, y limitaciones terapéuticas por el tipo de locación quirúrgica utilizada. Tabla 2.

La laparoscopia bajo anestesia local traza su concepción muy cercanamente con el desarrollo de la laparoscopia

Tabla 1 A

\section{VENTAJAS DE LA MICRO LAPAROSCOPIA} OFICINAL

\begin{tabular}{|l|}
\hline \multicolumn{1}{|c|}{ Paciente } \\
1. Disminución en la demora en la lista de espera \\
2. Disminución en la demora prequirúrgica \\
3. Eliminación de exámenes sanguíneos prequirúrgicos, \\
entrevistas y demoras \\
4. Reducción de los costos de cirugía \\
5. Disminución en la morbilidad postquirúrgica \\
6. Incremento en tasa de recuperación \\
7. Alta tasa de aceptación por el paciente
\end{tabular}

Tabla 1 B

VENTAJAS DE LA MICRO LAPAROSCOPIA OFICINAL

\begin{tabular}{|l|}
\hline \multicolumn{1}{|c|}{ Médico } \\
1. Disminución en la demora en la lista de espera \\
2. Disminución en la demora de los prequirúrgicos \\
3. Disminución en trabajo de escritorio \\
4. Reducción de los costos de cirugía \\
5. Eliminación de las demoras y el tiempo de viaje \\
\hline
\end{tabular}

Tabla $1 \mathrm{C}$

VENTAJAS DE LA MICRO LAPAROSCOPIA OFICINAL

\footnotetext{
2. Aumento en la tasa de recobro
}

Tabla 2

\section{DESVENTAJAS DE LA MICRO LAPAROSCOPIA OFICINAL BAJO ANESTESIA LOCAL}
1. Opciones terapéuticas limitadas
2. Campo de visión limitada
3. Tiempo quirúrgico limitado
4. Paciente no anestesiado
5. Paciente no dormido
6. Equipo frágil
7. Entrenamiento adicional es requerido
8. Costos iniciales

misma. Contrario a la creencia común, la laparoscopia se desarrolló inicialmente como un procedimiento bajo anestesia local, y no como un procedimiento con anestesia general (1). En una publicación realizada por Short en 1925 se describió por primera vez en Inglaterra la realización de la laparoscopia, así como su ejecución bajo anestesia local, en un sitio no tradicional para realizar un procedimiento quirúrgico (2).

En términos de seguridad, la laparoscopia comenzó como un procedimiento bajo anestesia local porque la anestesia general causa a los pacientes usualmente efectos colaterales y los mantiene indispuestos por varios días en el postoperatorio.

Al inicio de los años de 1970, varios cirujanos ginecólogos ensayaron promover la laparoscopia bajo anestesia local para la esterilización (3-6).

Con los pacientes bajo anestesia local estos doctores realizaron esterilizaciones tubáricas por laparoscopia usando corriente eléctrica, anillos para trompas y grapas. Estos procedimientos se realizaron en diferentes lugares, tales como sala de operaciones, sala de procedimientos de la consulta externa, y consultorios privados.

El movimiento de la laparoscopia bajo anestesia local ha sido acelerado colocándose en un primer plano de la laparoscopia, debido a las preocupaciones que generaron los altos costos del cuidado médico durante los años de 1980 y 1990.

Durante esta década, un número considerable de autores ha escrito acerca de los procedimientos realizados mediante la laparoscopia oficinal bajo anestesia local (7-18).

La micro laparoscopia se refiere a la inspección de los órganos internos por medio de endoscopios de calibre pequeño. En general, el término de "micro laparoscopio" se refiere a aquellos telescopios e instrumentos accesorios de un diámetro menor de $5 \mathrm{~mm}$ y recientemente, este término se ha reservado para instrumentos de $2 \mathrm{~mm}$. o menos en diámetro.

La primera generación de micro laparoscopios fue creada hace 20 años y adolecía de una óptica de mala calidad, la visualización estaba comprometida no permitiendo una completa evaluación de la pelvis.

Estos catéteres ópticos fueron sin embargo los precursores de los hoy en día laparoscopios fibrópticos miniaturizados. 
Uno de los pocos estudios reportados usando esta primera generación de micro laparoscopios fue escrito por Steege en 1994, quien realiza procedimientos bajo anestesia local usando la técnica de laparoscopia oficinal. Utilizando el mismo laparoscopio con la técnica de una sola punción él realizo lisis de adherencias recién formadas, reportando una alta tasa de éxito en la mejoría de síntomas en pacientes con historia de dolor pélvico crónico, así como una disminución en la reformación de adherencias después laparoscopias múltiples de segunda mirada (19).

\section{Indicaciones y selección de pacientes}

En términos generales la laparoscopia bajo anestesia local está indicada primariamente para diagnóstico y procedimientos quirúrgicos simples. Los procedimientos que son de la esfera de acción laparoscopia oficinal se describen en la tabla 3.

En ginecología, los únicos procedimientos en que existe información de tolerancia y eficacia son la laparoscopia diagnóstica para evaluación del dolor pélvico crónico, laparoscopia diagnóstica para evaluación de infertilidad y ligadura de trompas uterinas. Otros procedimientos se deben considerar de investigación en el momento actual. Las indicaciones quirúrgicas han sido expresamente limitadas a disminuir el riesgo de complicaciones relacionadas con los procedimientos.

La selección de pacientes para la laparoscopia oficinal es similar a la de los casos de laparoscopia diagnóstica regular en los que, tras una valoración del paciente mediante una historia clínica, exploración física y pruebas auxiliares correspondientes a los síntomas presentes, es preciso realizar una visualización directa de la cavidad peritoneal para apoyar él diagnóstico en curso (20-21).

Los criterios de exclusión son similares a los de la laparoscopia general, pero debe evitarse realizar este procedimiento en pacientes con historia de cirugía abdominal mayor tal como histerectomía, operación cesárea, apendicetomía y laparotomía exploradora, (en manos expertas, las contraindicaciones pueden ser relativas) también se deben excluir pacientes cuyo peso sea mayor de 100 kilos y aquellos con problemas médicos mayores con la anestesia.
Los pacientes obesos hacen más difícil la laparoscopia bajo anestesia local. La obesidad mórbida hace más difícil la inserción del trocar y aumenta la posibilidad de insuflación e inserción preperitoneal. Muchos cirujanos, utilizan una aguja de Veress con una camisa especial la cual es utilizada como trocar. Por tanto en caso de pacientes con obesidad mórbida puede necesitarse una aguja extra larga cuando se utiliza equipo miniaturizado.

La Sociedad Americana ha establecido un sistema de calificación que permite estratificar el riesgo anestésico del paciente con base en las enfermedades sistémicas subyacentes. Pacientes en la calificación I y II, la mayoría de ellos presenta enfermedades sistémicas leves, siendo esto los casos seleccionados para realizar laparoscopia oficinal.

Esta graduación disminuye el riesgo de anestesia y la tensión relacionada con las complicaciones (cardíacas y respiratorias principalmente) y simplifica los requerimientos de monitoría en el procedimiento.

Quizás el más importante requerimiento para el éxito de la laparoscopia bajo anestesia local es la propia determinación del paciente. Se debe discutir con el paciente todos los aspectos del procedimiento para evitar sorpresas y disminuir la ansiedad, se recomienda una comunicación verbal continua con el paciente, durante la colocación de la anestesia local y durante la realización del procedimiento, permitiendo que el paciente participe de toda la acción, observando el monitor del video al mismo tiempo que le estamos hablando.

\section{Técnicas}

\section{Instrumentación}

Los laparoscopios tradicionales están construidos de una serie de lentes de vidrio rígidos o lentes en varilla ensamblados entre sí. Estos están circundados por una camisa metálica, incorporan haces de fibra óptica para conducir la luz. Los laparoscopios de este tipo tienen un diámetro que varía de $5 \mathrm{~mm}$ a $12 \mathrm{~mm}$ y pueden incluir un canal operatorio.

Refinamientos en la tecnología de fibra óptica han permitido la creación de laparoscopios en los cuales el

Tabla 3

USOS POTENCIALES DE LA MICRO LAPAROSCOPIA BAJO ANESTESIA LOCAL

\begin{tabular}{|l|l|}
\hline \multicolumn{1}{|c|}{ Ginecología } & \multicolumn{1}{c|}{ Cirugía General } \\
\hline 1. Laparoscopia Diagnóstica & Evaluación del abdomen agudo \\
2. Evaluación de la infertilidad: & Evaluación del trauma abdominal \\
Cromopertubación & Herniorrafía \\
3. Evaluación del dolor pélvico: mapeo & Biopsia dirigida \\
consciente del dolor & Evaluación del dolor pélvico crónico \\
4. Lisis de adherencias & Diagnóstico de apendicitis \\
5. Tecnología en reproducción asistida & aguda \\
GIFT/ZIFT/TET & \\
6. Laparoscopia de segunda mirada & \\
7. Evaluación en oficinas de emergencia & \\
8. Ligadura de trompas & \\
\hline
\end{tabular}


elemento óptico está constituido por haces de cables de fibra óptica fundidos. Estos telescopios modernos de fibra óptica pueden tener de 10.000 a 30.000 fibras ópticas de imagen así como haces adicionales para transportar la luz. Los telescopios de fibra óptica de este tipo tienen un rango de 25 a 27 centímetros de longitud y un campo de visión de $60^{\circ}$ a $75^{\circ}$.

Los micro laparoscopios actuales tienen una visión directa de $0^{\circ}$, y su diámetro externo varía de $1 \mathrm{~mm}$ a $4 \mathrm{~mm}$. Ciertos micro laparoscopios incorporan una camisa rígida a la cual se fijan para permitir una inserción directa, lo cual aumenta su diámetro externo real.

Hemos observado una disminución dramática de la calidad y agudeza de la imagen con telescopios de un diámetro menor de $2 \mathrm{~mm}$. Dependiendo del diseño, estos laparoscopios de fibra óptica pueden ser igualmente durables y fuertes como los telescopios rígidos

Actualmente tenemos a disposición instrumental de $2 \mathrm{~mm}$ de diámetro para micro laparoscopia. En nuestra experiencia, los accesorios más usados son un palpador de punta roma y una pinza. Con las micro pinzas hay que tener cuidado al utilizarlas, pues sus pequeñas quijadas pueden trasmitir una gran fuerza al tejido y producir laceración de éste al ser aplicada en forma incorrecta la pinza. Existen también micro tijeras, sin embargo a la fecha todas son reusables y pierden su filo con el mínimo uso.

La gran limitación en instrumental de micro laparoscopia es la fuente de energía para hemostasia. En la actualidad hay varios prototipos de equipos para corriente unipolar o bipolar, pero su tamaño pequeño limita su utilidad, cuestionándose la seguridad y el costo beneficio del procedimiento en una oficina.

Además de un laparoscopio (miniaturizado o de tamaño estándar), se requiere el siguiente equipo para la laparoscopia oficinal.

1. Video cámara y monitor

2. Fuente de luz

3. Instrumental auxiliar y de equipo de acceso

4. Mesa que de posición de Trendelenburg

5. Equipo de resucitación

6. Equipo para aplicación intravenosa de medicación

7. Ayudante quirúrgico entrenado así como una persona encargada de monitorizar los signos vitales de la paciente, y de administrar sedación intravenosa.

\section{Anestesia}

Tradicionalmente se ha pensado que la laparoscopia es un procedimiento que requiere anestesia general. Muchos argumentos se han dado para sustentar esto.

1. La anestesia general se requiere para un control adecuado del dolor intra operatorio.

2. La creación del neumoperitoneo compromete el desplazamiento del diafragma y obliga a la intubación para una ventilación mecánica, para asegurar una adecuada ventilación.

3. Es necesaria para paralizar el paciente y así evitar movimientos intra operatorios y lesiones inadvertidas.

4. Es necesaria para realizar una ventilación mecánica que permita limpiar el CO2 absorbido del neumoperitoneo.
Todos los argumentos anteriores son basados en anécdotas y especulaciones y no en datos reales.

La realidad es que hay una historia larga que sustenta la seguridad, factibilidad, y ventajas de la laparoscopia bajo anestesia local que refutan los argumentos anteriores (22-26).

La mayoría de los procedimientos bajo anestesia local que ha sido reportado fueron realizados con algún grado de sedación intravenosa (IV) adicional a la anestesia local (19,22-23, 27).

En estos reportes, el término de anestesia local es usado liberal mente para diferenciar el procedimiento de aquellos realizados bajo anestesia general, aún si son complementados con agentes intravenosos (IV). La consideración principal del uso de sedación intravenosa consciente es la capacidad del paciente de estar alerta y de dar respuesta. Cuando se hace esto, el paciente podrá en forma independiente conservar la integridad de su propia vía aérea y mantener una ventilación adecuada. En contraste, con la anestesia general, hay una pérdida parcial o total de los reflejos protectores, incrementándose dramáticamente el riesgo de los procedimientos.

La laparoscopia se puede realizar usando estrictamente anestesia local; sin embargo, ésta resulta en una disminución de tolerancia en la medida que se aumenta el tiempo quirúrgico.

Históricamente, laparoscopia bajo estricta anestesia local (usando instrumentos de tamaño tradicional) se ha limitado a un tiempo quirúrgico de 10 a 15 minutos antes de que el paciente comience a presentar molestias que limiten el procedimiento.

\section{Premedicación y anestesia local}

Una vez se decide realizar una laparoscopia oficinal, la enfermera escolta a la paciente al sitio designado para realizar el procedimiento $y$ hace que se coloque sobre la mesa de cirugía en posición de litotomía dorsal, con sus pantorrillas sobre los estribos de la mesa quirúrgica, luego se canaliza una vena periférica con una aguja No. 18, conectada a una Dextrosa en solución salina al 5\%. (La línea intravenosa no se usa de rutina a menos que se desee para una sedación adicional. Al mismo tiempo la enfermera coloca a la paciente todos los elementos para medir presión arterial, cables para ECG y pulso oximetro.

A cada paciente se le da una premedicación intramuscular antes del procedimiento, la cual incluye atropina y $0,4 \mathrm{mg}$ y diazepam, 2,5mg

La anestesia local incluye 1) un bloqueo de un campo periumbilical utilizando una combinación de bupivacaina (marcaina) 10ml, y lidocaína (xilocaína) al 1\%, 10ml. 2) un bloqueo paracervical usando lidocaína al $1 \%$.

El bloqueo paracervical disminuye la posibilidad de reacción vasovagal.

$\mathrm{Si}$ al paciente se le va a realizar ligadura tubárica, a cada tuba individualmente se debe rosear antes con 5 ml de lidocaína al $1 \%$.

El cirujano realiza la parte abdominal y el asistente la parte vaginal simultáneamente. El asistente prepara la 
vulva y vagina con una solución de yodo y drena la vejiga, realiza el bloqueo paracervical.

\section{Bloqueo paracervical}

El bloqueo consiste en inyectar la solución anestésica alrededor del tronco nervioso, a cierta distancia de la zona que se desea anestesiar. Para ello, se debe pinzar el labio posterior con unas pinzas de Pozzi e inyectar a nivel de la inserción vaginal del labio posterior a $1,5 \mathrm{~cm}$ de la línea media, de cada lado, exactamente en el lugar de implantación de los ligamentos útero sacros, la aguja no debe profundizarse mas de $2 \mathrm{~cm}$, inyectándose de 5-6ml en cada lado esperando de 3-4 minutos para alcanzar su efectividad (28) Fig. 1.

\section{Bloqueo de la pared abdominal}

Utilizando la mezcla de anestésico descrito antes se infiltra la piel inmediatamente debajo del ombligo, para 10 cual utilizamos una aguja de pequeño calibre, luego de esto cambiamos a una aguja número 20 ó 22 para infiltrar la pared abdominal que levantamos con la mano. La infiltración se inicia en un ángulo de 45 grados con relación con el ombligo y se debe dirigir hacia el útero, luego en un trayecto hacia la izquierda en 45 grados se inyectan de 3 a $5 \mathrm{ml}$, y luego hacia la derecha en un ángulo de 45 grados se inyectan otros 3 a $5 \mathrm{ml}$ de anestésico, de esta forma se establece una área anestesiada que bloquea los nervios de la pared abdominal en el sitio de inserción del trocar asegurando que el procedimiento sea confortable (21) Fig. 2.

La experiencia le permitirá al médico determinar qué tan profunda debe ir la aguja y qué tanta cantidad de anestésico debe dejar en cada nivel.

\section{Procedimientos específicos}

Tanto la laparoscopia bajo anestesia local como la micro laparoscopia requieren de una gran pericia. No es suficiente tener una excelente trayectoria en laparoscopia avanzada para tener éxito es esta nueva técnica por tanto recomendamos a los nuevos practicantes en este campo seguir un programa de educacional formal.

Nosotros realizamos la laparoscopia oficinal bajo anestesia local en una oficina dedicada a procedimientos. El equipo de video y de insuflación esta localizado en un carro tradicional de una sala de cirugía. Colocamos dos mesas adyacentes al paciente, una para el equipo vaginal y otra para el abdominal. Al paciente colocado en posición de litotomía dorsal, le preparamos la vagina y perineo con una solución antiséptica. Después realizamos el bloqueo paracervical e insertamos el manipulador uterino (cánula de Rubin).

El abdomen es preparado con una solución antiséptica, cubriendo el área quirúrgica con campos estériles. Después hacemos un bloqueo del área periumbilical. Esto permite la inserción de un trocar de insuflación sobre la aguja de Veress. Esta aguja de Veress pasa a través del centro de la camisa, la cual incluye un puerto de insuflación. El conjunto aguja de Veress más camisa, es insertada de igual forma que la aguja estándar de Veress.
Luego el abdomen es insuflado con $\mathrm{CO} 2$ hasta crear una ventana visual adecuada. En total insuflamos de 0.5 a 2 litros aunque en algunos casos hemos llegado hasta 6 litros.

Un segundo trocar de $2 \mathrm{~mm}$ es insertado en la línea media a $4 \mathrm{~cm}$ por encima del pubis.

Figura 0017i01

INFILTRACION PARACERVICAL

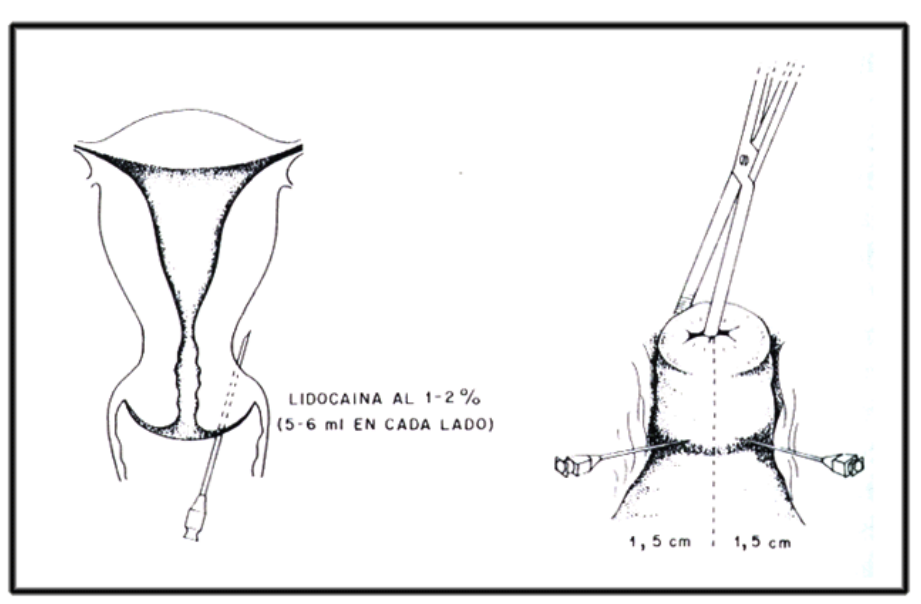

Figura 0017i02

BLOQUEO DE LA PARED ABDOMINAL

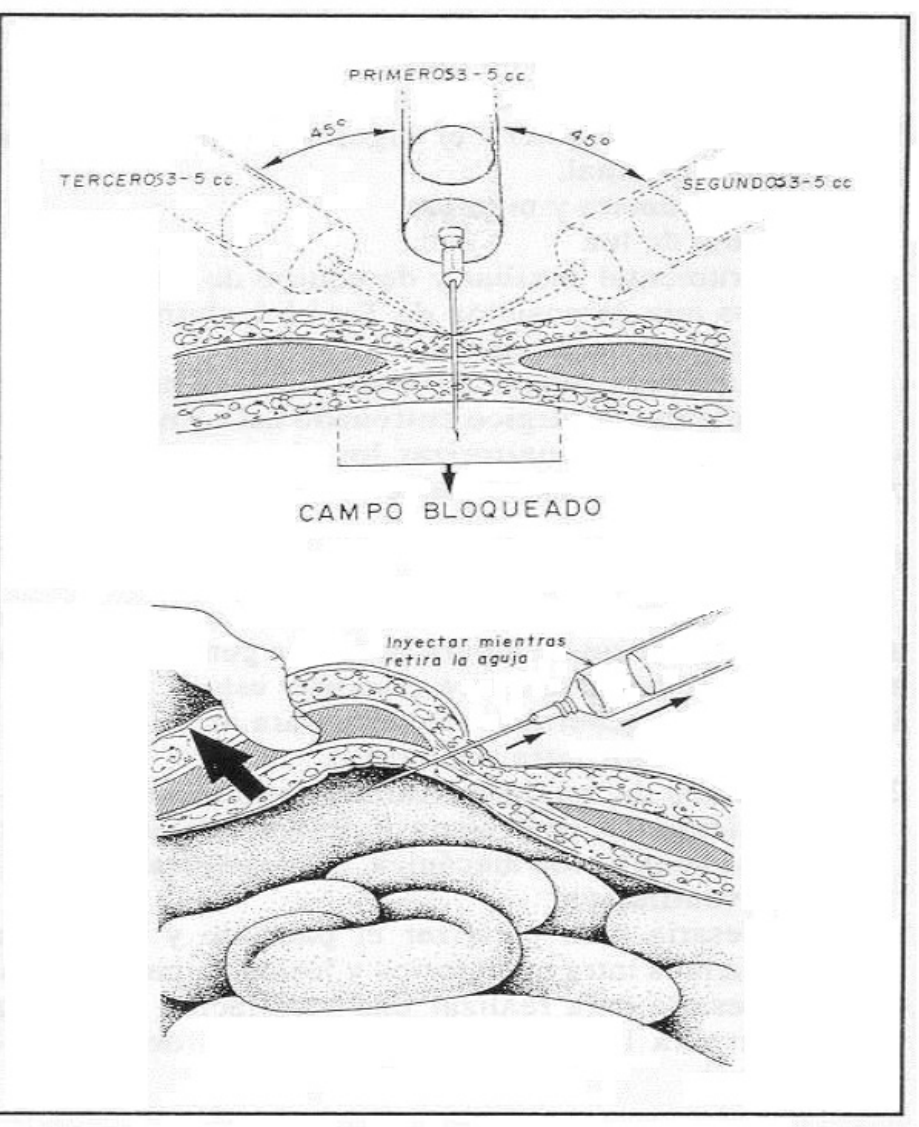


Se debe tener gran cuidado al insertar este trocar bajo visión directa, porque la punta de este puede pasar muy cerca de vísceras y vasos pélvicos.

Durante el curso de la operación el paciente es colocado en posición de Trendelenburg para ayudar a desplazar el intestino del fondo de saco de Douglas. En general $10^{\circ}$ a $25^{\circ}$ es suficiente.

$\mathrm{Si}$ se experimentan molestias excesivas internas, se pueden inyectar anestésicos directamente en la cavidad pélvica. Sin embargo hay que tener cuidado de no administrar grandes volúmenes que puedan causar toxicidad sistémica. Nosotros seguimos las dosis recomendadas por peso corporal que podría ser usada para la inyección de cualquiera de esos agentes.

Los instrumentos de tamaño tradicional también pueden ser utilizados para realizar la laparoscopia oficinal bajo anestesia local. Su uso, sin embargo es potencialmente más peligroso por el mayor grado de trauma que podría resultar de una lesión del trocar.

Similarmente nosotros hemos encontrado un incremento significante en comodidad ( $\mathrm{y}$ un aumento en la tolerancia a un mayor tiempo quirúrgico) al utilizar instrumentos miniaturizados. Utilizamos trocares e instrumentos para todos los procedimientos oficinales de segunda punción de $2 \mathrm{~mm}$, con excepción de la ligadura de trompas.

\section{Resultados}

\section{Esterilización tubárica bajo anestesia local}

La mayoría de los informes sobre laparoscopia bajo anestesia local fueron publicados en 1970 con el desarrollo de los métodos de esterilización por laparoscopia. Muchas de las técnicas para ligadura de trompas por laparoscopia se desarrollaron inicialmente bajo anestesia local usando los laparoscopios tradicionales de 10 y $12 \mathrm{~mm}$ con canal operatorio (21-25).

P. V, Metha realizó una extraordinaria serie de 250.000 ligaduras de trompas en un período de 9 años usando anillos de silastic (29). Los procedimientos fueron realizados en el área rural de la India. Simples bancos de madera colocados sobre bloques para producir posición de Trendelemburg fueron utilizados como mesas de cirugía. A pesar de este montaje, y el utilizar agua hervida para esterilizar los equipos, los resultados y complicaciones fueron similares a los de Occidente.

El autor reporta 74 casos de sangrado de la pared abdominal $(0.03 \%), 4$ casos de trauma intestinal requiriendo posterior laparotomía $(0.002 \%)$, y 12 muertes dentro de los primeros 30 días después del procedimiento $(0.005 \%)$, no como causa del procedimiento. No hubo reporte de lesiones vasculares mayores. La tasa de falla en el procedimiento fue del $0.1 \%$ y la incidencia total de complicaciones mayores que requirieron hospitalización fue del $0.003 \%$.
Hoy día, la esterilización laparoscópica bajo anestesia local generalmente se realiza vía a la tradicional técnica de una

punción o vía al método de dos punciones. La esterilización oficinal con dos punciones, se inserta un trocar secundario de

$5 \mathrm{~mm}$ a $4 \mathrm{~cm}$., por encima del pubis, realizando la aplicación de las grapas o anillos de silastic o coagulación de la trompa.

Recientemente hemos incorporado el uso de micro endoscopios para la realización de la esterilización tubárica con lo cual hemos aumentado la comodidad para la paciente.

Laparoscopia oficinal para evaluación de la
infertilidad

Se ha demostrado que se puede realizar una evaluación completa de la infertilidad mediante la laparoscopia oficinal con anestesia local, incluyendo la cromopertubación con azul de metileno, biopsia de endometriosis, inspección de todas las áreas de la pelvis y del abdomen. (27) La mayoría de los pacientes (96\%) reportaron gran aceptación del procedimiento. El promedio de tiempo del procedimiento fue 18 minutos con un rango de 8 a 50 minutos. Además las pacientes estuvieron en capacidad de dejar el sitio del procedimiento en menos de una hora, retornando a sus actividades normales dentro de las 24 horas siguientes. El análisis de costo demostró una reducción del $75 \%$ al compararla con la laparoscopia tradicional. Estos resultados sugieren que el uso de la micro laparoscopia oficinal puede ser considerada en la investigación temprana de la infertilidad. Dentro de este montaje, la selección adecuada del paciente es vital para reducir la posibilidad de que una intervención quirúrgica pueda ser requerida.

\section{Posibilidades futuras}

Se debe hacer una cuidadosa evaluación de esta nueva tecnología puesta en uso. Es imperativo, que no únicamente digamos que se puede hacer, si no que evaluemos críticamente esta nueva tecnología en términos de eficacia, costo efectividad, tolerancia y aceptación. Sin duda hay procedimientos quirúrgicos que no pueden ser realizados bajo anestesia local. Por ejemplo la laparoscopia diagnóstica oficinal se puede realizar con una técnica que permita una completa visualización de todas las áreas de la pelvis, las cuales se podrían visualizar igualmente con la laparoscopia tradicional. Igualmente una apendicetomía oficinal debe demostrar que es igualmente de ventajosa cuando se compara con el acceso laparoscópico tradicional para ser una opción viable. El peligro con la rápida proliferación de nueva tecnología (especialmente cuando hay incentivos económicos para preferirla) es aceptar el procedimiento sobre la base de anécdotas sin una documentación objetiva de eficacia y seguridad. Debe ser meta de aquellos quienes corrientemente desarrollan estas tecnologías asegurar la progresión de laparoscopia oficinal bajo anestesia local de una novedad a una norma ginecológica. 


\section{BIBLIOGRAFIA}

1. Gunning JE. The history of laparoscopy. J Reprod Med. 1974; 12; 222226.

2. Short AR. The use of celioscopy. Br. Med J. 1925; 2: 254-257.

3. Alexander G, Goldraht M, Brown E M et al. Outpatient laparoscopic sterilization under local anesthesia. Am J Obstet Gynecol 1973; 116: 1065-1070.

4. Fishburne P J. Office laparoscopic sterilization with local anesthesia. J Reprod Med. 1977; 18: 223-228.

5. Hulka J, Fishburne J, Mercer J. et al. Laparosocopic sterilization with a spring clip: A report of the first fifty cases. Am J Obstet Gynecol 1973; 116: 715-720.

6. Penfield A. Gynecologic Surgery under Local Anesthesia. Baltimore. Munich, Urban and Schwarzeburg 1986; 189.

7. Bordahl, Raeder, Nordentoft, et al. Laparoscopic sterilization under local anesthesia or general anesthesia? A randomized study. Obstet Gynecol. 1993; 81: 137-141.

8. Childers J, Hatch K, Surwit E et al. Office laparoscopy and biopsy evalutation of patients with intraperitoneal carcinomatosis using a new optical catheter. Gynecol Oncol. 1992; 47: 337-342.

9. Friedrich P J. Outpatient laparoscopy under local anesthesia in a procedure room. In First Master Course in Office Laparoscopy under Local Anesthesia. Proceedings of the American Association of Office Endoscopy. May 3-5. 1996. Tuskegu AL. Tuskegu University. P. 1-8.

10. Lipscomb G, Sumwit R, McCord M, et al. The effect of nitrous oxide and carbon dioxide premoperitoneam on operative and post operative pain during laparoscopic sterilization under local anesthesia. J Am Assoc Gynecol Laparosc. 1994; 2: 57-60.

11. Love B, McCorvey, R McCorvey M. Low cost office laparoscopic sterilization. J Am Assoc Gynecol Laparosc. 1994; 1: 379-384.

12. McCorvey R, Love B. Microendoscopic office laparoscopy under local anesthesia. Second International Symposium and Hands - on Workshop on office Micro Endoscopy and Endoscopic Microsurgery in Gynecology. San Francisco, CA, The American Association of Gynecologic Laparoscopy. Jul 19-21, 1996 p. 80.

13. Metha P. Laparoscopic sterilization with Falope ring: Experience with 10.100 women in rural camps. Obstet Gynecol. 1981; 57: 345-350.
14. Palter S. Moving laparoscopy into the office: OBG. Management 1955 : 9: 27-36.

15. Penfield A: Twenty two years of office and outpatient laparoscopy: Current techniques and why I chose them. J Am Assoc Gynecol Laparosc, 1995; 2: 365-368.

16. Poindexter A, Abdul - Malak M, Fast J. Laparoscopic tubal sterilization under local anesthesia. Obstet Gynecol 1990; 75: 5-9

17. Risquez F, Pennhouar O, Fernández R et al. Microlaparoscopy: A prelirninary report, Human Repro. 1993; 8: 1701-1702.

18. Steege J F. Repeated clinic laparoscopy for the treatment of pelvic adhesions: A pilot study, Obstet Gynecol. 1994; 83: 276-279.

19. Gomel V, Taylor P K. Diagnostic and Operative Gynecologic Laparoscopy, 1995, Mosby Year Book Inc., p: 68-70.

20. Hulka J, Reich H. Text Book of Laparoscopy. Phyladelphia, WB Saunders. 1985; p. 81-83, 136-151.

21. Peterson H B, Hulka J F, Spielman F J, Lee S, Marchbanks PA. Local vs general anesthesia for laparoscopic sterilization: Randornized study. Obstet Gynecol, 1987; 70: 903-908.

22. Brown D R, Fishburne JI, Roberson VO, Hulka JV. Ventilatory and blood gas changes during laparoscopy with local anesthesia. Am J Obstet Gynecol. 1976; 124: 741-745.

23. Wheeles CR Jr. Outpatient laparoscopic sterilization under local anesthesia. Obstet Gynecol 1972; 39: 767-770.

24. Alexander G D, Goldrath M, Brown E M, Smiler BG. Outpatient laparoscopic sterilization under local anesthesia. Am J Obstet Gynecol 1993; 116: 1065-1068.

25. Palter S, Olive D. Office laparoscopy under local anesthesia for chronic pelvic pain: Utility, acceptance, and cost-benefit analysis. J Am Assoc Gynecol Laparosc 1996; 3: 359-364.

26. Palter SF, Olive DL. Office laparoscopy under local anesthesia for infertility: Utility, acceptance, and cost - benefit/ outcome analyser. Fertil Steril 1995; 64: S8-S9.

27. Labastida R N. Tratado y Atlas de Histeroscopia. Salvat Editores, S.A. Barcelona, 1990; 28-30.

28. Metha PV. A total of 250.136 laparoscopic sterilizations by single operator. Br J Obstet Gynecol 1989; 96: 1024-1034. 